(C) 2007 IEEE. Personal use of this material is permitted. Permission from IEEE must be obtained for all other uses, in any current or future media, including reprinting/republishing this material for advertising or promotional purposes, creating new collective works, for resale or redistribution to servers or lists, or reuse of any copyrighted component of this work in other works. 


\title{
Application of Multi-level Multi-domain Modeling in the Design and Analysis of a PM Transverse Flux Motor with SMC Core
}

\author{
Youguang Guo*, Jianguo Zhu*, Dikai Liu*, Haiyan Lu**, and Shuhong Wang*** \\ * Faculty of Engineering, University of Technology, Sydney, NSW 2007, Australia \\ ** Faculty of Information Technology, University of Technology, Sydney, NSW 2007, Australia \\ *** State Key Laboratory of Electrical Insulation and Power Equipment, Faculty of Electrical Engineering, \\ Xi'an Jiaotong University, Xi'an, 710049, China
}

\begin{abstract}
This paper presents the design and analysis of a permanent magnet (PM) transverse flux motor with soft magnetic composite (SMC) core by applying multi-level multi-domain modeling. The design is conducted in two levels. The upper level is composed of a group of equations which describe the electrical and mechanical characteristics of the motor. The lower level consists of two domains: electromagnetic analysis and thermal calculation. The initial design, including structure, materials and major dimensions, is determined according to existing experience and empirical formulae. Then, optimization is carried out at the system level (the upper level) for the best motor performance by optimizing the structural dimensions. To successfully deal with such a multi-level multi-domain optimization problem, an effective modeling with both high computational accuracy and speed is required. For accurately computing the key motor parameters, such as back electromotive force, winding inductance and core loss, magnetic field finite element analysis is performed. The core loss in each element is stored for effective thermal calculation, and the winding inductance and back EMF are stored as a look-up table for effective analysis of the motor's dynamic performance. The presented approach is effective with good accuracy and reasonable computational speed.
\end{abstract}

Index Terms-Motor design, multi-level multi-domain modeling, permanent magnet (PM) transverse flux motor, soft magnetic composite (SMC) material.

\section{INTRODUCTION}

The design of electrical motors is a complex task involving many aspects such as performance prediction, parameter calculation, dimension and structure optimization, mechanical and thermal calculation, etc. A large amount of models and approaches have been developed by various researchers for these analyses and computations. For example, parameters can be computed with empirical formulae or magnetic field finite element analysis (FEA). The empirical formulae, based on many simplifications and assumptions, often produce large computational error and cause incorrect prediction of the motor performance, although the simulation speed is impressive. Any incorrect simulation may cause design failure and expensive design changes [1]. On the other hand, the approach based on numerical magnetic field

This work was partly supported by the Australian Research Council Discovery Project Grants (DP0773858). analysis can provide high computational accuracy, but the CPU time can be very long. The long computational time is a big problem for the design and analysis of a complex device such as an electrical machine, which is composed of multi-levels and multi-domains. Therefore, an effective modeling with both high accuracy and high speed is always desired by motor designers.

With the help of effective multi-level multi-domain modeling, this paper presents the design and analysis of a transverse flux motor (TFM) with soft magnetic composite (SMC) core and permanent magnet (PM) flux concentrating rotor. The core material, SOMALOY ${ }^{\mathrm{TM}}$ 500, is a relatively new SMC material developed by Höganäs AB, Sweden [2]. SMC materials possess many unique properties like magnetic isotropy and very low eddy current loss and hence are very suitable for applications of electrical machines with complex structures and three-dimensional (3-D) fluxes, such as claw pole motors and TFMs [3-4]. In a TFM, the magnetic field at the armature has significant component along any direction. Consequently, 3-D numerical analysis should be conducted to accurately determine the field distribution and key motor parameters.

A hierarchical two-level modeling approach is applied for the design of this TFM, as illustrated in Fig. 1. The upper level consists of a group of electrical and mechanical equations, which describe the motor characteristics. The lower level includes two domains: electromagnetic design and thermal calculation. Electromagnetic design is to mainly determine key motor parameters such as PM flux (defined as the flux of one phase winding produced by rotor PMs), back electromotive force (EMF), winding inductance and core loss, based on magnetic field FEAs. Thermal calculation is to predict the temperature rises of key locations such as winding and PMs, which must be within the limits for safe operation of the motor.

The initial design, including the structure, materials and dimensions, is determined based on existing experience and empirical formulae. Then, optimization is conducted at the system level for the best motor performances, such as the highest torque to cost ratio within a certain volume. To effectively couple the designs at different levels and different domains, the core loss at each element from electromagnetic design is 
stored as the input of thermal calculation, and the patterns of back EMF and winding inductance from electromagnetic analysis are stored for the motor performance prediction at the upper level. By this way, a good compromise between accuracy and speed can be achieved.

Another important domain, mechanical design, can be included in the lower level. In this paper, the mechanical strength is guarantied by our experience, e.g. the minimal core dimension is controlled to be no less than $6 \mathrm{~mm}$ during the optimization process. It should be noted that more domains and levels, such as the mechanical design, could be easily added, leading to a more complex design architecture.

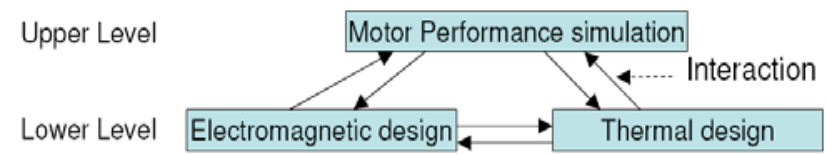

Fig. 1. Two-level hierarchical motor design architecture

\section{PM TRANSVERSE FluX MOTOR WITH SMC CORE}

PM machines with transverse flux structure have attracted strong interest of research since Weh et al. proposed the first versions of TFMs in the 1980s [5]. Capable of producing very high specific torque provided that the number of poles is large, TFMs are naturally suitable for direct drive applications which request large torque at low rotational speed.

Generally, TFMs have complex structure with a large number of components, especially for the double-sided stator type, so it is very difficult to manufacture the cores by using the conventional laminated steels. TFMs have large leakage flux, which is 3-D in nature, in addition to the 3-D main flux. The flux component perpendicular to the lamination plane may cause excessive eddy currents. These problems have greatly limited the applications of TFMs, but they may be overcome by the development of new SMC materials [3-4]. The applications of SMCs in TFMs have been investigated by several researchers, and the results are quite promising [6-8].

Based on our previous experience with SMC motors [4, 8-9], this paper presents the design and analysis of a three-phase three-stack TFM with modified double-sided stator and PM flux concentrating rotor. SMC is used as the cores of both stator and rotor. Fig. 2 illustrates the magnetically relevant parts of one pole-pair of one stack (the single concentrated coil is not shown for clarity). Each stack forms a phase and three phases are axially stacked with the stator cores shifted by $120^{\circ}$ electrical and the rotor PMs aligned. The rotor is supported by cantilevers on the shaft.

The main dimensions and parameters of the TFM include: $100 \mathrm{~mm}$ for the stator outer diameter, $56 \mathrm{~mm}$ for the stator and rotor inner diameter, $33 \mathrm{~mm}$ for the effective axial length of each stack, $9.5 \mathrm{~mm}$ for the PM radial length, and 20 poles. The motor is designed to operate with a brushless DC (BLDC) control scheme, delivering a power of $280 \mathrm{~W}$ at $3000 \mathrm{rev} / \mathrm{min}$ for compressor driving.

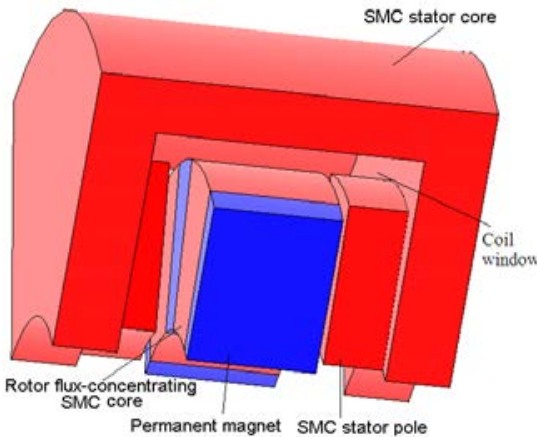

Fig. 2. Structure and FEA solution region of one pole-pair of one stack of a TFM

\section{ELECTROMAGNETIC DESIGN}

Electromagnetic analysis is conducted to determine the motor's key parameters. Due to the complex structure and 3-D field distribution, analytical and empirical formulae cannot provide accurate computation. Therefore, numerical field analysis like FEA is required, allowing the nonlinear properties of the materials and structural details to be considered. Because of the magnetic independence between stacks and the symmetry of the motor structure, it is only required to analyze the magnetic field in one pole-pair region of one phase, as shown in Fig. 2. At the two radial boundary planes, the magnetic scalar potential used to solve the magnetic field distribution obeys the so-called periodical boundary conditions:

$\varphi_{m}(r, \Delta \theta, z)=\varphi_{m}(r,-\Delta \theta,-z)$

where $\Delta \theta=30^{\circ}$ is the angle of one pole pitch. The original point of the cylindrical coordinate is located at the center of the motor.

From the numerical field distribution, many key motor parameters can be accurately obtained. For example, from the no-load field solutions, the curve of PM flux against rotor angle is found to be an almost perfect sinusoid with magnitude of $\phi_{1}=0.358 \mathrm{mWb}$, so the back EMF constant is calculated as $0.232 \mathrm{Vs} / \mathrm{rad}$ by

$$
K_{E}=\frac{p}{2} N_{s} \frac{\phi_{1}}{\sqrt{2}}
$$

where $p=12$ is the number of poles and $N_{s}=153$ is the number of turns of a phase winding.

With the numerical magnetic field solutions at no-load, the cogging torque can be calculated by using the virtual work method or Maxwell stress tensor method. It is found that this TFM has a negligible cogging torque with a peak value of $0.02 \mathrm{Nm}$, comparing to the rated output torque of $0.89 \mathrm{Nm}$. The major cogging torque component of one stack is the fundamental, which is cancelled by those of the other two stacks.

Winding inductance is another key parameter determining the motor performance. When the magnetic circuit is saturated, the motor performance depends on the incremental (differential) inductance rather than the secant (apparent) inductance [10]. In this paper, the winding inductance of the TFM is computed by a 
modified incremental energy method [11], which consists of the following steps: (1) Conduct a nonlinear analysis with the excitations of both rotor PMs and stator currents; (2) Calculate and save the incremental permeability in each element; (3) Conduct a linear analysis with the saved permeability and the excitation of a perturbed stator current $\Delta i$ (from zero current) only; (4) Calculate the magnetic co-energy $\Delta W_{c}$. Then, the winding incremental inductance can be obtained by

$$
L_{i n c} \approx \frac{2 \Delta W_{c}}{(\Delta i)^{2}}
$$

Because each phase has an almost independent magnetic circuit, the mutual inductances between phase windings can be considered as zero. The average self incremental inductance of one phase winding is computed as $13.0 \mathrm{mH}$.

Unlike conventional electrical machines in which the copper loss is the dominant component of total power loss, an SMC machine has comparable core loss and copper loss, so accurate prediction of core loss is important in the motor design and analysis. It has been long known that core losses are caused by not only alternating but also rotating magnetic fields, and core losses caused by different patterns are very different [12]. In the TFM, when the rotor rotates, the flux density loci at certain location can be alternating (one-dimensional) with or without harmonics, circularly or elliptically rotating within a two-dimensional plane which may not be parallel to any axis, or even an irregular loop in a real 3-D space.

An improved method is applied for predicting the core losses of this 3-D flux SMC motor [13]. Different formulations are used for core loss prediction with alternating, circularly rotating, and elliptically rotating flux density vectors, respectively. The core loss of the TFM is computed as $16.3 \mathrm{~W}$ at $3000 \mathrm{rev} / \mathrm{min}$. Due to the very small eddy current loss, the total core loss can be considered proportional to the rotor speed, or operational frequency.

Other parameters determined include: phase winding resistance of $2.304 \Omega$, copper loss of $15.6 \mathrm{~W}$, and mechanical loss of $8.8 \mathrm{~W}$.

\section{THERMAL DESIGN}

Thermal design is crucial for the economical utilization of materials and safe operation of a motor. Commonly used methods for thermal analysis of electrical machines include lumped parameter thermal model [14], thermal network and thermal FEA [15]. The thermal network combines the accuracy of FEA and the speed of lumped parameter thermal model. As the core loss distribution has been obtained in each element, which can be directly input as the heat sources of thermal analysis, thermal FEA and a hybrid thermal model [16] are employed to analyze the temperature rise of the TFM. During the optimization process, the maximum temperatures of winding and $\mathrm{PM}$ are controlled as 139.5 ${ }^{\circ} \mathrm{C}$ and $120{ }^{\circ} \mathrm{C}$, respectively.

\section{Motor PERformance Simulation}

\section{A. Steady-State Characteristics}

The motor can be operated with a BLDC drive scheme. At synchronous motor mode, the steady-state characteristic of the TFM can be predicted by an equivalent electrical circuit, as shown in Fig. 3, where $E_{1}$ is the root-mean-squared (RMS) value of induced back EMF, $R_{1}$ the phase winding resistance, $\omega_{1}$ the operational angular frequency, and $L_{1}$ the synchronous inductance, which equals the self inductance of a phase winding plus half the mutual inductance between two phase windings. All these parameters have been obtained in the Electromagnetic Design.

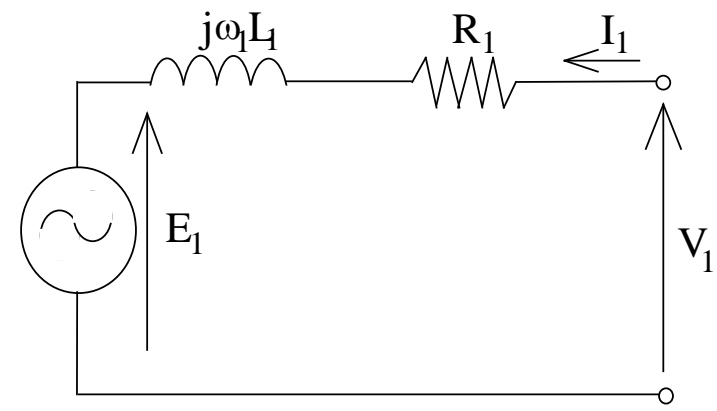

Fig. 3. Per-phase equivalent electrical circuit of PM synchronous motor

At the optimum BLDC control condition, the stator current $I_{1}$ is in phase with $E_{1}$, so that the electromagnetic power and torque can be calculated by

$$
P_{e m}=m E_{1} I_{1}
$$

$T_{e m}=\frac{P_{e m}}{\omega_{r}}=K_{T} I_{1}$

where $\omega_{r}$ is the rotor speed in mechanical rad/s, $K_{T}=m K_{E}$ is the torque constant, and $m=3$ is the number of phases. The RMS value of the back EMF is obtained by $E_{1}=K_{E} \omega_{r}$.

For a given terminal voltage, $V_{1}$, the characteristic of rotor speed against electromagnetic torque can be derived as

$$
\omega_{r}=\frac{\sqrt{\left(\frac{R_{1} T_{e m}}{m}\right)^{2}+\left[\left(\frac{p}{2} L_{1} \frac{T_{e m}}{K_{T}}\right)^{2}+K_{E}^{2}\right]\left[V_{1}^{2}-\left(\frac{R_{1} T_{e m}}{K_{T}}\right)^{2}\right]}-\frac{R_{1} T_{e m}}{m}}{\left(\frac{p}{2} L_{1} \frac{T_{e m}}{K_{T}}\right)^{2}+K_{E}^{2}}
$$

The output power $P_{\text {out }}$, output torque $T_{\text {out }}$, input power $P_{\text {in }}$ and efficiency $\eta$ of the total drive system can be calculated by

$$
\begin{aligned}
& P_{\text {out }}=P_{\text {em }}-P_{\mathrm{Fe}}-P_{\text {mec }} \\
& T_{\text {out }}=P_{\text {out }} / \omega_{r} \\
& P_{\text {in }}=P_{\text {em }}+P_{\text {inv }}+P_{c u} \\
& P_{c u}=3 I_{1}^{2} R_{1}
\end{aligned}
$$




$$
\eta=P_{\text {out }} / P_{\text {in }}
$$

where $P_{F e}$ is the core loss, $P_{m e c}$ the mechanical loss, $P_{i n v}$ the inverter conduction loss, $P_{c u}$ the copper loss, and $\omega_{r}$ the rotor angular speed in mechanical rad/s.

\section{B. Dynamic Performance}

The dynamic characteristics of the motor are determined by a group of equations as

$$
\begin{aligned}
& v_{j}=r_{j} i_{j}+d \lambda_{j} / d t+e_{j}, \quad j=a, b, c \\
& \lambda_{j}=\sum_{k=a}^{c} L_{j k} i_{k} \\
& T_{e m}=\left(\sum_{j=a}^{c} e_{j} i_{j}\right) / \omega_{r}+T_{c o g} \\
& J \frac{d \omega_{r}}{d t}=T_{e m}-T_{L}-\delta \omega_{r} \text { and } \frac{d \theta_{r}}{d t}=\omega_{r}
\end{aligned}
$$

where all the variables are of their conventional meanings.

For the symmetrical $\mathrm{Y}$-connected 3 phase windings without central line, we have

$$
\begin{aligned}
& i_{a}+i_{b}+i_{c}=0 \\
& r_{a}=r_{b}=r_{c} \\
& L_{a b}=L_{b a}, L_{b c}=L_{c b}, L_{c a}=L_{a c}
\end{aligned}
$$

Based on (12)-(18), a complete simulation model can be built in Simulink environment [17]. This model can be applied to analyze the steady and dynamic performances of BLDC motors to predict if the design requirements can be met, e.g. the dynamic performance of start-up, i.e. whether or not the motor can reach the required steady speed under the full load when the rated DC voltage of the inverter is applied.

An optimization routine for the design of the PM transverse flux motor with SMC core has been set up, which considers multi-levels and multi-domains. The magnetic field FEAs, thermal analysis, performance calculation, and optimization searches are all implemented in a commercial comprehensive software package, ANSYS. It is found that the presented method is practical with both good accuracy and speed.

\section{CONCLUSIONS AND DISCUSSIONS}

In this paper, a permanent magnet transverse flux motor with soft magnetic composite core is designed and analyzed by using a two-level modeling. The lower level consists of electromagnetic design domain and thermal analysis domain. The upper level consists of the performance simulation domain only and at this level optimization is carried out in close interaction with the lower level. Appropriate modeling is applied to achieve effective interaction between various levels and domains, with both good computational accuracy and speed.

The design and analysis of a motor drive is a complex task. For a practical drive system, many aspects such as motor type and topology design, power electronic circuit design, controller system design, and dimension and loading design and material selection, should also be considered [18]. It should be noted that these aspects could be easily added to the hierarchical motor design architecture of Fig. 1, leading to a more complex multilevel multi-domain design problem.

\section{REFERENCES}

[1] B. Knorr, D. Devarajan, D. Lin, P. Zhou, and S. Stanton, "Application of multi-level multi-domain modeling to a claw pole alternator," in Proc. Annual Conf. of Society of Automotive Engineers, USA, 2004, Paper 2004-01-0758.

[2] "Soft magnetic composites from Höganäs Metal Powders SOMALOY' 500,” Höganäs Product Guide, 1997.

[3] "The latest development in soft magnetic composite technology," SMC Update, Reports of Höganäs $A B$, Sweden, 1997-2007. At http://www.hoganas.com/, see News then SMC Update.

[4] Y. G. Guo, J. G. Zhu, P. A. Watterson, and W. Wu, "Comparative study of 3-D flux electrical machines with soft magnetic composite core,” IEEE Trans. on Industry Applications, vol. 39, no. 6, pp. 1696-1703, Nov. 2003.

[5] H. Weh and H. May, "Achievable force densities for permanent magnet excited machines in new configuration," in Proc. Int. Conf. on Electrical Machines, Munich, Germany, 1986, pp. 1107-1111.

[6] B. C. Mecrow, A. G. Jack, and C. P. Maddison, "Permanent magnet machines for high torque, low speed applications," in Proc. Int. Conf. on Electrical Machines, Vigo, Spain, 1996, pp. 461-466.

[7] G. Henneberger and M. Bork, "Development of a transverse flux traction motor in a direct drive system," in Proc. Int. Conf. on Electrical Machines, Helsinki, Finland, 2000, pp. 1457-1460.

[8] Y. G. Guo, J. G. Zhu, P. A. Watterson, and W. Wu, "Development of a permanent magnet transverse flux motor with soft magnetic composite core," IEEE Trans. on Energy Conversion, vol. 21, no. 2, pp. 426-434, June 2006.

[9] Y. G. Guo, J. G. Zhu, P. A. Watterson, and W. Wu, "Development of a claw pole permanent magnet motor with soft magnetic composite stator," Australian $J$. Electrical \& Electronic Eng., vol. 2, no. 1, pp. 21-30, 2005.

[10] M. Gyimesi and D. Ostergaard, "Inductance computation by incremental finite element analysis," IEEE Trans. Magn., vol. 35, no. 3, pp. 1119-1122, May 1999.

[11] Y. G. Guo, J. G. Zhu, and H. Y. Lu, "Accurate determination of parameters of a claw pole motor with SMC stator core by finite element magnetic field analysis," IEE Proceedings - Electric Power Application, vol. 153, no. 4, pp. 568-574, July 2006.

[12] Y. G. Guo, J. G. Zhu, J. J. Zhong, and W. Wu, “Core losses in claw pole permanent magnet machines with soft magnetic composite core,” IEEE Trans. Magn., vol. 39, no. 5, pp. 3199-3201, Sep. 2003.

[13] Y. G. Guo, J. G. Zhu, Z. W. Lin, and J. J. Zhong, "Measurement and modeling of core losses of soft magnetic composites under 3D magnetic excitations in rotating motors,” IEEE Trans. Magn., vol. 41, no. 10, pp. 3925-3927, Oct. 2005.

[14] P. H. Mellor, D. Roberts, and D. R. Turner, "Lumped parameter thermal model for electrical machines of TFFC 
design,” Proc. Inst. Elect. Eng. Pt. B, vol. 138, no. 5, pp. 205-218, Sep. 1991.

[15] G. Cannistrà, G. Cannistrà, and M. S. Labini, "Thermal analysis in an induction machine using thermal network and finite element methods," in Proc. IEE EMD Conf., 1991, pp. 300-304.

[16] Y. G. Guo, J. G. Zhu, and W. Wu, "Thermal analysis of SMC motors using a hybrid model with distributed heat sources,” IEEE Trans. Magn., vol. 41, no. 6, pp. 21242128, June 2005.
[17] Y. G. Guo, J. G. Zhu, J. X. Chen, and J. X. Jin, "Performance analysis of a permanent magnet claw pole SMC motor with brushless DC control scheme," in Proc. Int. Power Electronics and Motion Control Conf., Shanghai, China, 13-16 Aug. 2006, Vol. 2. pp. 1-5.

[18] J. G. Zhu, D. K. Liu, and Y. G. Guo, “Application oriented system level optimum design method for advanced electrical drive systems," Australian Research Council Discovery Project, DP0773858, 2007-2009. 